\title{
Compact Assembly and Programmable Integration of Supercapacitors
}

\author{
Bing Lu, Feng Liu, Guoqiang Sun, Jian Gao, Tong Xu, Yukun Xiao, Changxiang Shao, \\ Xuting Jin, Hongsheng Yang, Yang Zhao, Zhipan Zhang, Lan Jiang, and Liangti Qu*
}

Microsized supercapacitors ( $\mathrm{mSCs}$ ) with small volume, rapid charge-discharge rate, and ultralong cyclic lifetime are urgently needed to meet the demand of miniaturized portable electronic devices. A versatile self-shrinkage assembling (SSA) strategy to directly construct the compact mSCs (CmSCs) from hydrogels of reduced graphene oxide is reported. A single $\mathrm{CmSC}$ is only $0.0023 \mathrm{~cm}^{3}$ in volume, which is significantly smaller than most reported $\mathrm{mSCs}$ in fiber/yarn and planar interdigital forms. It exhibits a high capacitance of up to $68.3 \mathrm{~F} \mathrm{~cm}^{-3}$ and a superior cycling stability with $98 \%$ capacitance retention after 25000 cycles. Most importantly, the SSA technique enables the $\mathrm{CmSC}$ as the building block to realize arbitrary, programmable, and multi-dimensional integration for adaptable and complicated power systems. By design on mortise and tenon joint connection, autologous integrated 3D interdigital CmSCs are fabricated in a self-holding-on manner, which thus dramatically reduces the whole device volume to achieve the high-performance capacitive behavior. Consequently, the SSA technique offers a universal and versatile approach for large-scale on-demand integration of $\mathrm{mSCs}$ as flexible and transformable power sources.
mSCs with effectively increased capacitance and decreased device volume remains challenging.

Over the past decade, various techniques such as photolithography, ${ }^{[6,7]}$ laser-direct writing and laser etching, ${ }^{[8,9]}$ printing and spray coating ${ }^{[10-12]}$ have been successfully used to fabricate $\mathrm{mSCs}$ in fiber/yarn-based or planar interdigital forms. ${ }^{[13-17]}$ Despite of these achievements, they suffer some inherent drawbacks. For instance, photolithography and printing methods are usually complicated and less powerful in constructing adaptable and transformable mSC systems. Meanwhile, the size and volume of interdigital $\mathrm{mSCs}$ obtained by laser-direct writing considerably increased after the integration of current collectors, supporting substrates, and packing materials, ${ }^{[18-20]}$ a scenario that even became worse when a large number of mSCs were connected in series/parallel for practical applications. ${ }^{[21-24]}$

With the rapid development of miniaturized and portable electronic devices, microsized supercapacitors (mSCs) with small space volume, rapid charge-discharge rate, and ultralong cyclic lifetime have captured enormous attention. ${ }^{[1-3]}$ While the areal capacitance of mSCs has been well improved recently, their practical use is still hindered by the low volumetric capacitance resulted from the low packing density. ${ }^{[4,5]}$ Therefore, fabricating
In this work, we report a straightforward and versatile selfshrinkage assembling (SSA) strategy to directly fabricate compact $\mathrm{mSC}(\mathrm{CmSC})$. Aided by the surface tension of water during evaporation, the CmSC is spontaneously fabricated during the shrinkage of reduced graphene oxide (rGO) hydrogel slices with a layer of graphene oxide (GO) as the separator and conductive wires (e.g., gold, carbon fiber) as current collectors. The whole
Dr. B. Lu, Dr. G. Sun, Dr. J. Gao, Dr. T. Xu, Y. Xiao, Dr. C. Shao,

Dr. X. Jin, Dr. H. Yang, Prof. Y. Zhao, Prof. Z. Zhang, Prof. L. Qu

Key Laboratory of Photoelectronic/Electrophotonic Conversion Materials

Key Laboratory of Cluster Science

Ministry of Education of China

School of Chemistry and Chemical Engineering

Beijing Institute of Technology

Beijing 100081, P. R. China

E-mail: lqu@bit.edu.cn

Prof. F. Liu

State Key Laboratory of Nonlinear Mechanics

Institute of Mechanics

Chinese Academy of Sciences

Beijing 100190, P. R. China

The ORCID identification number(s) for the author(s) of this article can be found under https://doi.org/10.1002/adma.201907005.
Prof. L. Jiang

Laser Micro-/Nano-Fabrication Laboratory

School of Mechanical Engineering

Beijing Institute of Technology

Beijing 100081, P. R. China

Prof. L. Qu

Key Laboratory for Adv. Mater. Processing Technology

Ministry of Education of China

State Key Laboratory of Tribology

Department of Mechanical Engineering

Tsinghua University

Beijing 100084, P. R. China

Prof. L. Qu

Department of Chemistry

Tsinghua University

Beijing 100084, P. R. China

DOI: 10.1002/adma.201907005 
device is compact and strong enough to lift heavy objects that are 5000 times of its own weight. A single CmSC features a low volume of only $0.0023 \mathrm{~cm}^{3}$ but an unprecedented capacitance of up to $68.3 \mathrm{~F} \mathrm{~cm}^{-3}$, setting up a new benchmark for carbonbased $\mathrm{mSCs}$ with gel electrolyte. Owing to the excellent plasticity of rGO hydrogels, the CmSC can be further shaped into various geometries. Most importantly, CmSCs work effectively as the building blocks for arbitrary integration to obtain $\mathrm{mSC}$ systems based on the SSA technique. Particularly, 3D interdigital CmSCs can be integrated into a more compact entity with higher power through the riveting of mortise and tenon joint structure. Therefore, the SSA strategy offers a universal approach for programmably constructing compact and highperformance mSCs based on hydrogel-type electrode materials.

The SSA strategy is used to prepare the CmSC. The rGO hydrogel was used as the initial material and synthesized via a two-step hydrothermal method (see the Experimental Section for details). The low temperature led to an initial reduction of GO and the sheets aggregated at a relatively high temperature. Compared with conventional hydrothermal method, the two-step hydrothermal treatment effectively restrained the fast and uneven aggregation of GO sheets, guiding the rGO hydrogel with more even inner structure (Figure S1a,b, Supporting Information). A computer-controlled laser engraving system was utilized to prepare the slices of rGO hydrogel block (Figure S1c, Supporting Information) and GO slurry was sandwiched between two rGO slices as separator and binder, together with inserted conductive wires (e.g., gold wires) as current collectors (Figure 1a). Thereinto, some adhesion-related applications have been demonstrated to use GO as glue. ${ }^{[25,26]}$ This kind of property is mainly related to the proper sheets size (several $\mu \mathrm{m})$, abundant surface, and edge-sited oxygenated a

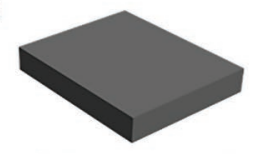

rGO hydrogel slice

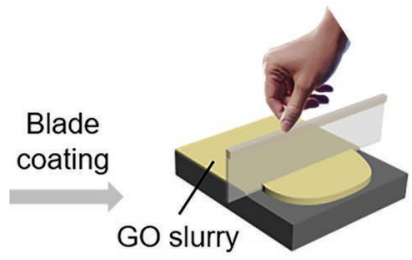

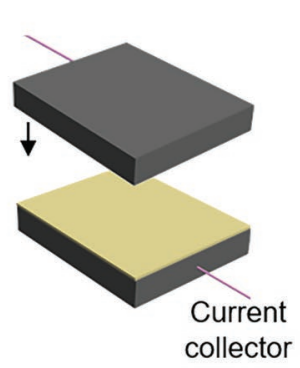

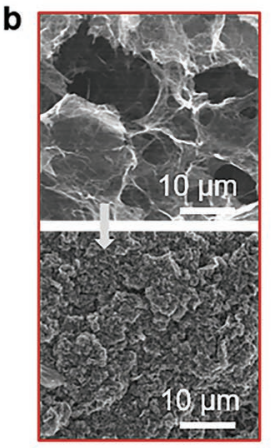

Shrinkage
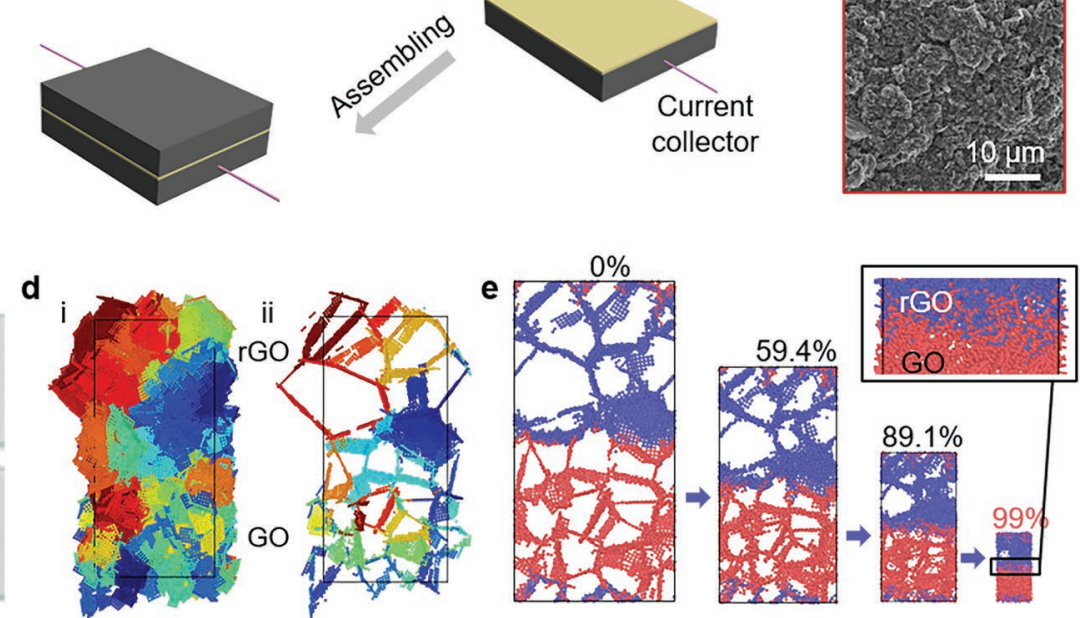

$\mathbf{f}$

C
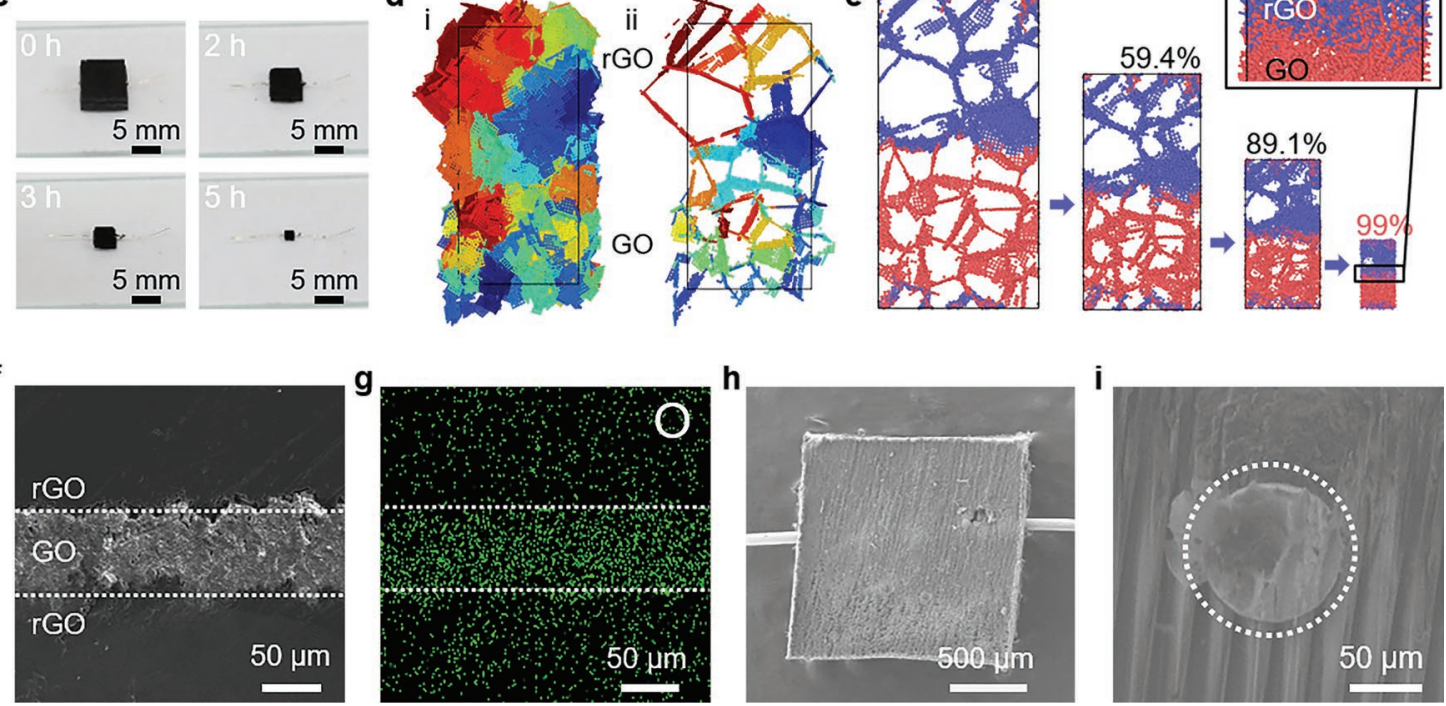

Figure 1. The fabrication and characterization of the CmSC. a) Schematic diagram of the fabrication of $\mathrm{CmSC}$. b) SEM images of rCO electrode before and after shrinkage. c) The shrinkage evolution process of $\mathrm{CmSC}$ from the initial status. d) Shrinkage simulation of the CmSC: initial configuration of $\mathrm{rGO} / \mathrm{GO}$ heterostructures. i) The side view of the initial configuration. ii) One slice of the initial configuration, showing that the generated configuration can well reproduce the porous structure. e) Deformation mechanism. Void evolution from calculation during the contraction is shown, and the corresponding four volume shrink ratios are $0 \%, 59.4 \%, 89.1 \%$, and $99 \%$, respectively. f) Cross-section SEM and g) the corresponding $O$ element mapping images of $\mathrm{CmSC}$ by EDX. h) SEM image of the prepared $\mathrm{CmSC}$. i) Cross-section SEM image of the implanted gold wire with rGO electrode. The circle part is gold wire. 
groups on GO sheets. The rGO hydrogel/GO/rGO hydrogel structure was left at the ambient condition and the subsequent dehydration led to a strong inward force on rGO sheets. ${ }^{[27]}$ As demonstrated in Figure 1b, the initial porous foam-like structure of rGO hydrogels gradually vanished and aggregation of rGO sheets was triggered by the surface tension of water during evaporation (Figure S2a, Supporting Information), making the hydrogel contract drastically to a high density of $1.49 \mathrm{~g} \mathrm{~cm}^{-3}$ (Figure 1c). Eventually, the dense rGO monolith had micropores with a size distribution of about $1-5 \mathrm{~nm}$ (Figure S2b, Supporting Information). The pore diameter is applicable to the aqueous, gel, and ionic liquid electrolytes. ${ }^{[28-30]}$

Coarse grain molecular dynamics (MD) simulation was performed to rationalize the microscale deformation mechanism during the SSA contraction. A $30 \times 30 \times 60 \mu \mathrm{m}^{3}$ region around the $\mathrm{rGO} / \mathrm{GO}$ interface was focused (Figure S3a, Supporting Information) and the detailed process of the simulation is presented in the Experimental Section. As shown in Figure 1d, the upper and bottom layers represent rGO and GO, respectively. During the simulated contraction, the size of the void gradually decreases with the decreasing volume (Figure 1e), as is consistent with experimental observations. By tracking 200 sheets during the contraction process, both inter-sheet adhesion and intra-sheet wrinkling are prominent and believed to significantly contribute to the deformation (Figure S4, Supporting Information). The energy evolution diagram shown in Figure S5 (Supporting Information) suggests most part of total energy for deformation comes from adhesion energy and angle energy (due to sheets wrinkling), implying the major role of sheets adhesion as well as wrinkling in deformation. More specifically, the adhesion energy (van der Waals energy) between rGO and GO sheets dominates in the beginning, and contraction induced by water evaporation helps to minimize the energy of the system. As contraction continues with sheets wrinkling, the angle energy rapidly increases and slightly combines contribution from bond energy (intra-sheet contraction) to eventually balance the adhesion energy gain and stop further contraction. Interestingly, GO slurry could dehydrate and shrink spontaneously due to the confinement by rGO hydrogel electrodes on top and bottom (Figure 1e). During the dehydration process, planar GO sheets tend to stack onto each other. ${ }^{[25]}$ On the other hand, surface and edge-sited oxygenated groups (e.g., carboxyl, carbonyl, and hydroxyl) of GO sheets make GO tightly attached to $\mathrm{rGO}$ sheets through hydrogen bonding, $\pi-\pi$ interactions, and van der Waals interactions, resulting in a strong sandwich structure for the CmSC (Figure 1f,g). In addition, the size of GO sheet $(3 \mu \mathrm{m})$ is smaller than the pore diameter $(12 \mu \mathrm{m})$ of initial rGO hydrogel. As the increase of shrink ratio, the part of GO sheets on the interface of GO slurry and rGO hydrogel were squashed in the pore of hydrogel and immobilized (enlarged view in Figure 1e). The tight coupling of electrode and separator makes the sandwich structure of $\mathrm{CmSC}$ stronger. Compared with initially assembled rGO hydrogel device, the CmSC underwent approximately fivefold reduction in linear dimensions and retained $1 \%$ of the volume, indicating an obvious densification process during the self-shrinkage formation of the CmSC (Figure 1h, Figure S6a, Supporting Information). The as-prepared CmSC was only $0.0023 \mathrm{~cm}^{3}$ in volume. During the shrinking process, gold wire was firmly embedded in the rGO electrode (Figure 1i), and thus the CmSC was strong enough to lift a heavy object that is 5000 times of its own weight (Figure S6b, Supporting Information). Smaller CmSCs can be fabricated via control over the size of rGO hydrogel slices through adjusting the laser cutting process (Figure S7, Supporting Information). Specially, the lateral dimension of the CmSC is less than $1 \mathrm{~mm}$, which should meet the requirement of the miniaturized and portable electronic devices.

Four CmSCs with different volume shrink ratios were denoted as $0 \% \mathrm{CmSC}$ (hydrogel state), $60 \% \mathrm{CmSC}$, $90 \% \mathrm{CmSC}$, and the typical CmSC (volume shrink ratio: 99\%). The volume shrink ratios were controlled by the dehydration time at room temperature. With the volume of the device decreased from 0.256 to $0.0023 \mathrm{~cm}^{3}$, the pore diameter of electrode materials shrunk from microns to nanometers (Figures S8 and S9, Supporting Information). Concomitantly, the volume-specific surface area of electrode materials drastically increased from 3 to $368 \mathrm{~m}^{2} \mathrm{~cm}^{-3}$ while only a slight change in mass-specific surface area was noticed. The invariability of mass-specific surface area demonstrates that no dead volume was formed during the dehydration process, suggesting there was no loss of specific surface area while the rGO sheets shrunk. As a result, the volumetric capacitance of the device increased from 0.6 for $0 \% \mathrm{CmSC}$ to $47 \mathrm{~F} \mathrm{~cm}^{-3}$ for typical $\mathrm{CmSC}$, respectively (Figure 2a,b and Figure S10, Supporting Information). Additionally, the electrochemical performance of $\mathrm{CmSCs}$ with different electrode thicknesses was also studied (see details in Figures S11 and S12, Supporting Information). In brief, the CmSC with smaller electrode thickness exhibited better capacitance and rate capability due to the easy transport of electrolyte ions. However, to achieve a higher specific energy at a single-device level, the moderate electrode thickness of $2 \mathrm{~mm}$ for hydrogel state and $450 \mu \mathrm{m}$ for the CmSC were executed in this work.

Figure 2c plots galvanostatic charge-discharge (GCD) profiles of a typical CmSC at different current densities of 50-800 $\mathrm{mA} \mathrm{cm}^{-3}$, with cyclic voltammetry (CV) curves at scan rates from 10 to $200 \mathrm{mV} \mathrm{s}^{-1}$ shown in Figure S13a (Supporting Information). Notably, the highly symmetric triangle shape in GCD curves and the nearly rectangular fashion at low scan rate in CV curves suggest typical electrical double-layer capacitive behaviors of rGO. ${ }^{[31,32]}$ Meanwhile, the IR drop in GCD profiles is relatively tiny, signifying an excellent effective voltage of the compact device. To explain the outstanding performance, electrochemical impedance spectroscopy (EIS) was further conducted to explore the kinetics of ion transport in CmSC (Figure S13c, Supporting Information). As revealed by the Nyquist plot, the CmSC shows a low equivalent series resistance of only $13.8 \Omega$, indicating fast ion diffusion at the interface of electrode materials and electrolyte. The volume-specific capacitance is calculated as $47 \mathrm{~F} \mathrm{~cm}^{-3}$ at a current density of $50 \mathrm{~mA} \mathrm{~cm}^{-3}$, which is better than that of other carbon-based mSCs with gel electrolyte reported previously (Figure $2 \mathrm{~d}$ ). ${ }^{[9,17,26,33-40]}$ The huge volume-specific surface area and good contact interface between electrode materials and electrolyte, electrode materials and current collector contributed to the outstanding electrochemical performance of $\mathrm{CmSC}$ together. Furthermore, under high-rate GCD cyclic measurement, the capacitance of the CmSC kept increasing during the initial cycles and remained stable after 3500th cycles (Figure 2e). Eventually, the CmSC showed a 

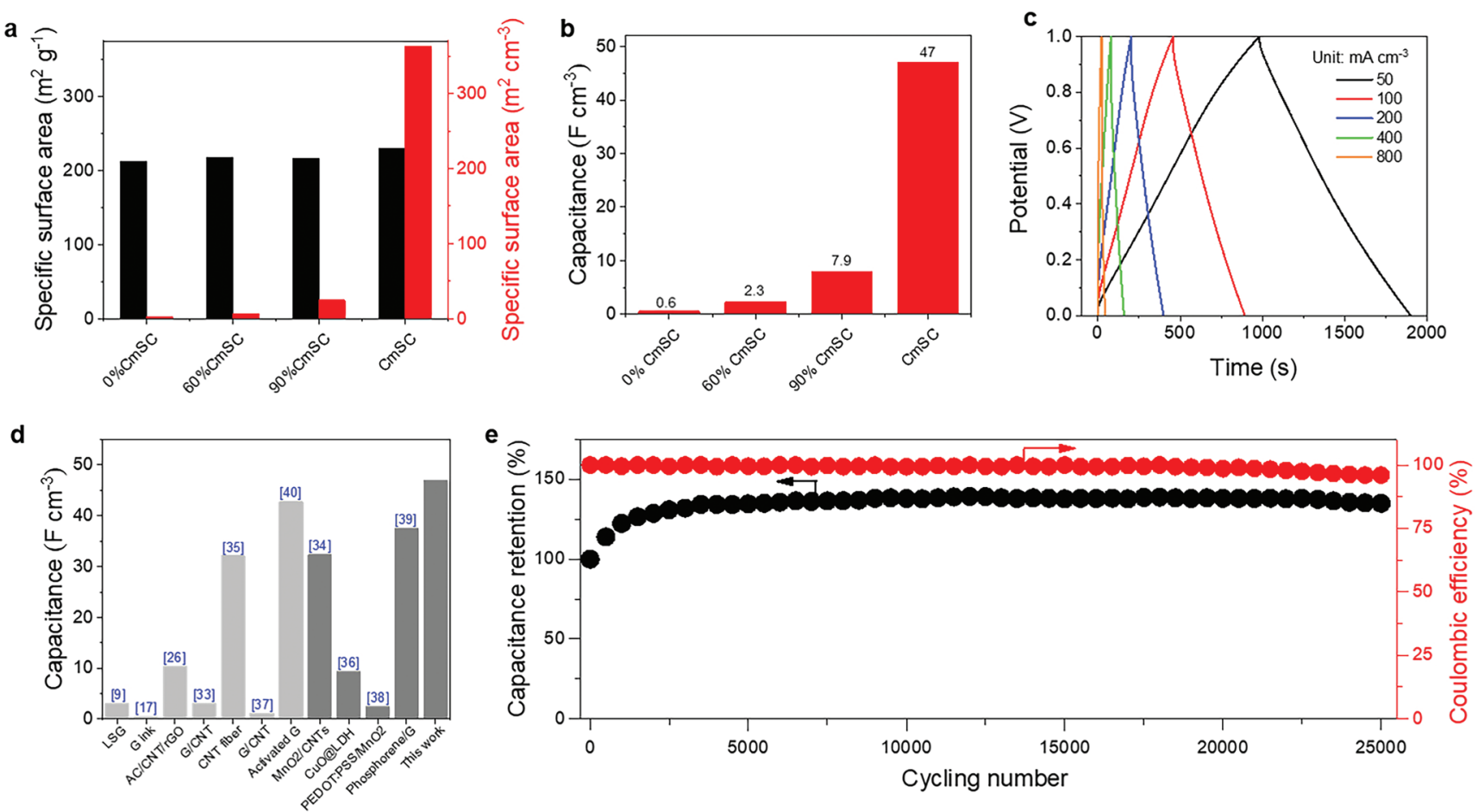

Figure 2. Electrochemical performance of $\mathrm{CmSC}$. a) Specific surface area of electrode materials in $\mathrm{CmSCs}$ with different volume shrink ratios. The specific surfaces of unit volume increase from 3 to $368 \mathrm{~m}^{2} \mathrm{~cm}^{-3}$, while the specific surfaces of unit mass show slight difference. b) Volume-specific capacitance comparison of $\mathrm{CmSCs}$ with different volume shrink ratios. c) GCD profiles of CmSC measured at different current densities ranging from 50 to $800 \mathrm{~mA} \mathrm{~cm}^{-3}$. d) Volumetric capacitance comparison of different mSCs. e) Cycling stability of $\mathrm{CmSC}$ for $25000 \mathrm{cycles}$, tested at $1.2 \mathrm{~A} \mathrm{~cm}^{-3}$.

remarkable cycling stability after 25000 cycles, retaining $135 \%$ of its initial capacitance, $98 \%$ of its steady capacitance, and a high Coulombic efficiency of $\approx 96 \%$. The initial increase of capacitance is attributed to the gradual deeper infiltration of the electrolyte into the dense electrode materials during the successive cyclic test and corresponding $\mathrm{Cl}$ element mapping image confirms the full infiltration of the electrolyte in this process as well (Figure S14c, Supporting Information). In addition, the sandwich structure and whole device volume remained original after the cyclic test (Figures S14a and S15, Supporting Information). These results mean that neither rGO nor GO would be swelled or destroyed with gel electrolytes during the performance evaluation process. We speculate that the rGO hydrogel after complete contraction is in the lowest energy state, and the process of shrinking is irreversible.

rGO hydrogel electrodes with different geometries could be designed and fabricated with the assistance of laser cutting (Figure S16, Supporting Information). After complete shrinkage, the constructed CmSCs retained their initial forms precisely (Figure 3a). The phenomenon indicated the inner uniformity of rGO hydrogel, which guaranteed the force equality for different part of the CmSC. In addition, as a representative of heteromorphic $\mathrm{CmSCs}$, the star-shaped CmSC exhibits a capacitance of $46.4 \mathrm{~F} \mathrm{~cm}^{-3}$ at $50 \mathrm{~mA} \mathrm{~cm}^{-3}$, with a capacitance retention of $89 \%$ at $200 \mathrm{~mA} \mathrm{~cm}{ }^{-3}$ and $47 \%$ at $800 \mathrm{~mA} \mathrm{~cm}^{-3}$, respectively (Figure $3 \mathrm{~b}$ ). In comparison, the square $\mathrm{CmSC}$ also showed a similar capacitance (Figure 3c), indicating an insensitivity of electrochemical performance to the shape change. Additionally, appropriately designed annular CmSCs could encircle small insects or birds to potentially power tracking chip (Figure S17a,b, Supporting Information). The annular $\mathrm{CmSC}$ was put on the wing before shrinkage and immobilized stably after shrinkage, reducing the volume of power supply and minimizing the influence of human interference to animal behaviors. For larger energy output, a number of annular CmSCs could be bunched in series (Figure S17c, Supporting Information). The integrated device possesses the potential as a portable power source.

Due to the versatility of the SSA method, it is also viable to fabricate 3D interdigital CmSCs that fully exploit the improved interface with fast ion transport. ${ }^{[40]}$ Herein, rGO electrodes based on mortise and tenon joint structure were prepared by laser cutting (Figure 4a,b, Figure S18, Supporting Information) and subsequently assembled to form a 3D interdigital $\mathrm{CmSC}$ with a comparable volume to a typical CmSC. Owing to the increased contact area between electrode material and electrolyte, GCD curves of the 3D interdigital CmSC exhibited longer charging-discharging time and a higher volumetric capacitance (Figure S19, Supporting Information). At a lower current density of $50 \mathrm{~mA} \mathrm{~cm}{ }^{-3}$, the volumetric capacitance of the $3 \mathrm{D}$ interdigital $\mathrm{CmSC}\left(68.3 \mathrm{~F} \mathrm{~cm}^{-3}\right)$ was higher than that of the normal CmSC at the same current density (Figure 4c), validating the concept of further exploiting fast ion transport for better electrochemical performance in the 3D interdigital CmSC. In addition, compared with the special CmSC, vertical contact surface of two rGO electrodes were added. Even so, no short circuit occurred in the device, which proves from another side that GO slurry as separator could attach well with the rGO 

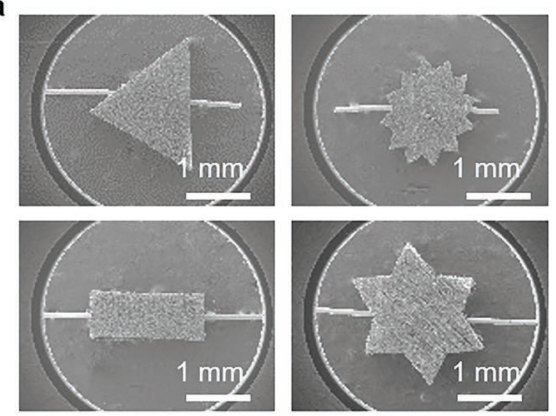

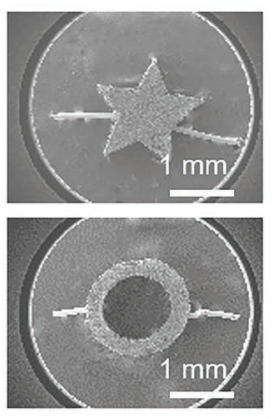

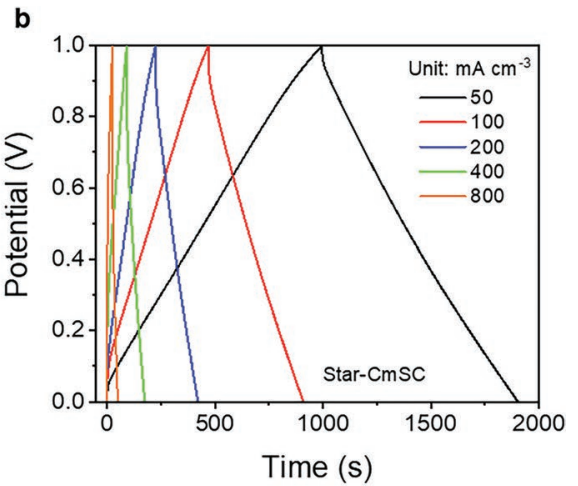

c

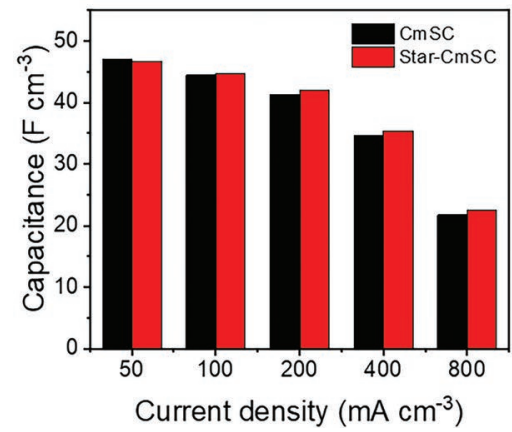

d

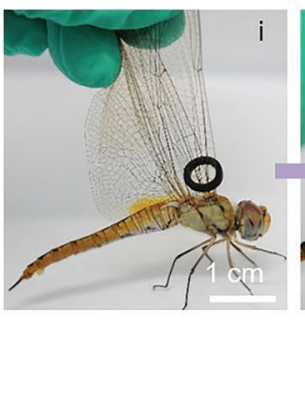

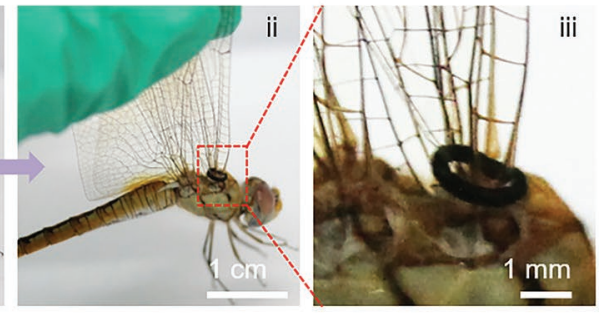

iii

Figure 3. Fabrication of CmSCs with arbitrary geometries. a) SEM images of shape-tailored CmSCs. b) GCD profiles measured at different current densities ranging from 50 to $800 \mathrm{~mA} \mathrm{~cm}^{-3}$ of star-CmSC. c) Volume-specific capacitance comparison of star-CmSC to square one. d) Digital images of an annular CmSC on dragonfly wing. i) Before shrinkage. ii) After shrinkage. iii) Corresponding enlarged view.

electrode. Apart from mortise-and-tenon style, the electrodes could also be shaped to other forms to increase the conjunction of two electrodes in 3D interdigital CmSCs (Figure S20, Supporting Information). For the further increase of contact area between electrode material and electrolyte, these new device designs show the potential to take better advantage of the electrode materials and achieve higher performance.

Following this idea, rGO hydrogel could be sculptured to a mortise-and-tenon joint structure on both upper and lower sides to fabricate an autologous integrated CmSC (AI-CmSC) consisting of series-connected electrodes in a self-holdingon manner (Figure 4d). Since the intermediate electrode is shared with two single CmSCs, the AI-CmSC actually removes the usage of any extra current collector and stands as small and rigid blocks after complete contraction (Figure 4e). Compared to that of the single $\mathrm{CmSC}$, the voltage of AI-CmSC doubles or triples as the integration number $(n)$ goes up from 1 to 2 (Figure $4 \mathrm{f}$ ) and a high voltage of $7 \mathrm{~V}$ was achieved when $n$ reached 6 . The AI-CmSC can easily power a motor fan for a long time (Figure 4g).

$\mathrm{CmSCs}$ can also be integrated in series or parallel configuration (I-CmSCs) by conventional current collectors (Figure 5a,b). For this purpose, two pairs of current collectors were introduced and electrochemical measurements indicated that the introduction of extra gold wires inside the electrode had no significant effect on device performance (Figure S21, Supporting Information). As plotted in Figure 5c and Figure S22 (Supporting Information), both CV and GCD profiles of series-connected I-CmSCs display a stepwise linear increase in the working voltage, implying the excellent performance uniformity of CmSCs.
Similarly, the current density in CV curves of parallel-connected I-CmSCs increases stepwise under the same voltage window, and the discharge time is also twice and three times longer than that of a single CmSCs under the same discharge current density. Benefiting from the small volume and outstanding performance, an I-CmSC bracelet was then prepared by encapsulating seven series-connected $\mathrm{CmSCs}$ in a flexible and air-tight heat shrinkable tube to reduce the evaporization loss of the electrolyte as well as the damage to the integrated device during kink (Figure 5d,e and Figure S23a, Supporting Information). It could power a blue light-emitting diode (LED) for a long time (Figure 5f). Furthermore, the CmSC could be packaged individually with colored sheath and a finger ring-type of CmSCs can also be easily prepared (Figure 5g and Figure S23b,c, Supporting Information). The configuration further improves the stability, practicality, and functionality of I-CmSCs.

Besides simple integration, networks of I-CmSC could also be fabricated by using CmSCs as building blocks (Figure S24a,c, Supporting Information). Under this scenario, I-CmSCs are arranged in multiple series-parallel connections and expected to improve the output of current and voltage simultaneously. In particular, the I-CmSC net shows considerable deformation tolerance during stretching or compression without any noticeable breakage (Figure S24b, Supporting Information). With the selection of proper kick points, the I-CmSC ball could be compressed compactly and completely restored to its initial shape through stretching (Figure S24d, Supporting Information). Due to the excellent flexibility of I-CmSC, it is also possible to transform the planar integration to multi-dimensional form (Figure 5h,i,k). With different angles of bending, an 
a

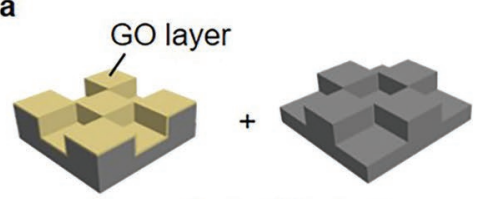

Assembling

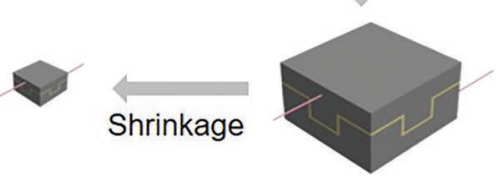

b

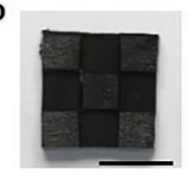

Electrode i

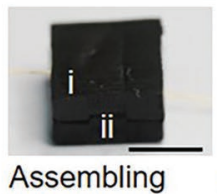

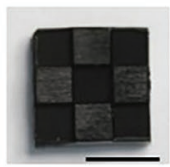

Electrode ii

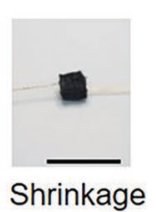

c

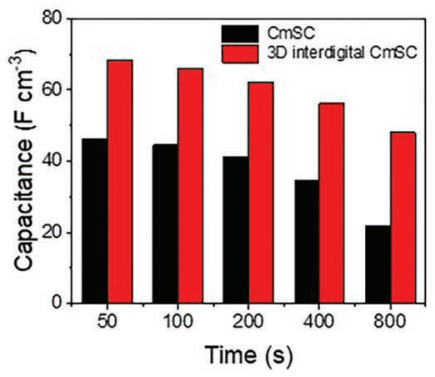

d
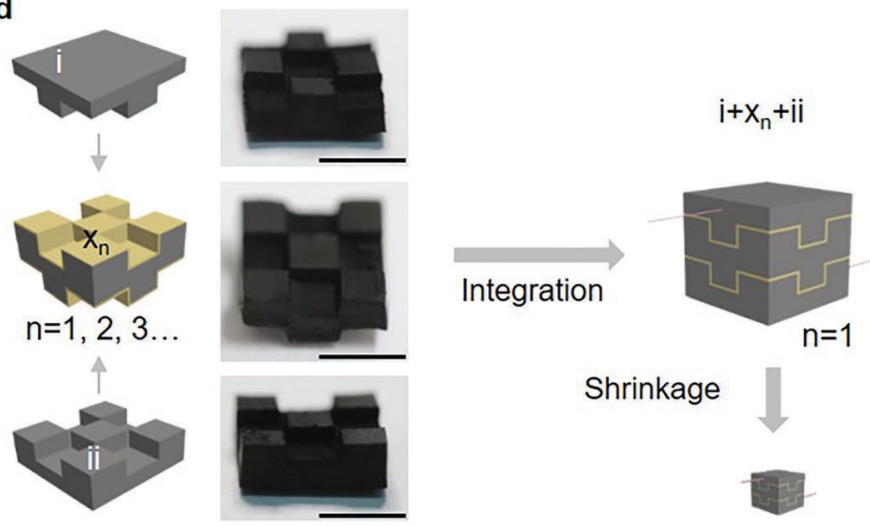

Multileve
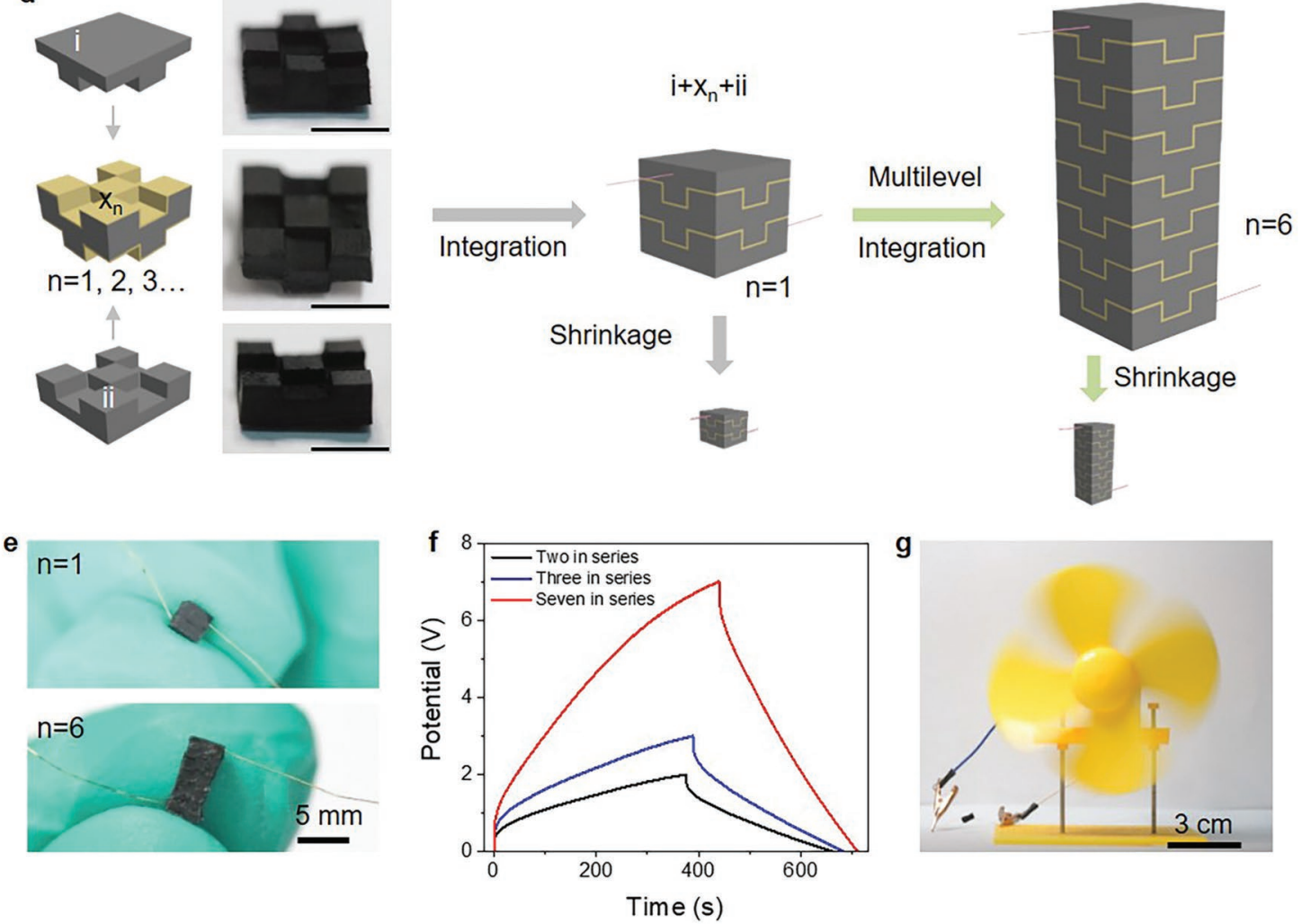

Figure 4. Fabrication and characterization of 3D interdigital CmSCs. a) Schematic diagram of the fabrication process for 3D interdigital CmSC. b) Optical images of 3D interdigital rGO electrodes and $\mathrm{CmSC}$ before and after shrinkage. Scale bars: $5 \mathrm{~mm}$. c) Volumetric specific capacitance comparison of 3D interdigital CmSC to normal one. d) Schematic diagram of the fabrication of autologous integrated CmSCs (Al-CmSCs). $n$ stands for the total number of the intermediate electrode. Scale bars: $5 \mathrm{~mm}$. e) Optical images of Al-CmSCs. f) GCD profiles tested at $200 \mathrm{~mA} \mathrm{~cm}^{-3}$ of Al-CmSCs with 2-3 and 7 cells connecting in series. g) Photograph of a motor fan powered by an Al-CmSC $(n=6)$.

as-fabricated planar I-CmSC starfish could be re-shaped to a sphere or a cone, where the sphere comprised six I-CmSC strings connected in parallel and each string contained three series-connected CmSCs. With a complementary red LED placed in the center of the sphere, a Chinese-style I-CmSC lantern was obtained (Figure 5j). Alternatively, the I-CmSC cone could be well fixed on the ribs of a mini-umbrella and the opening/closing of the umbrella triggered the on-off states of an embedded LED on the shaft (Figure 51 and Figure S24j, Supporting Information). Due to the high flexibility of the $\mathrm{I}-\mathrm{CmSC}$, the whole process had no damage to the device and this ensured long-term operation.
In this work, we have developed an SSA strategy to fabricate compact mSCs with rGO hydrogels as the starting material. A single CmSC device is only $0.0023 \mathrm{~cm}^{3}$ in volume and displays a volumetric capacitance of up to $68.3 \mathrm{~F} \mathrm{~cm}^{-3}$, the highest observed in a gel electrolyte for carbon-based mSCs. In addition, the CmSC exhibits superior cycling stability as well. Benefitting from the SSA technique, each of the CmSCs can be a building block for arbitrary integration and multidimensional construction of adaptable and complicated mSC systems. Most importantly, based on the mortise-and-tenon joint structure and connection, autologous integrated 3D interdigital CmSCs is proposed and fabricated. The AI-CmSC dramatically reduces 


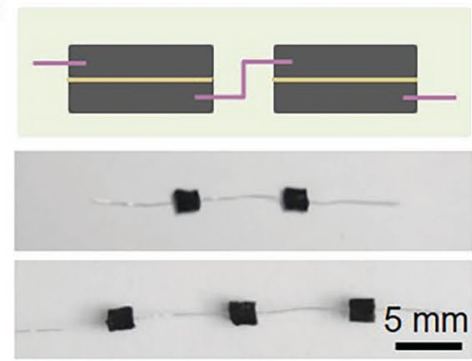

b

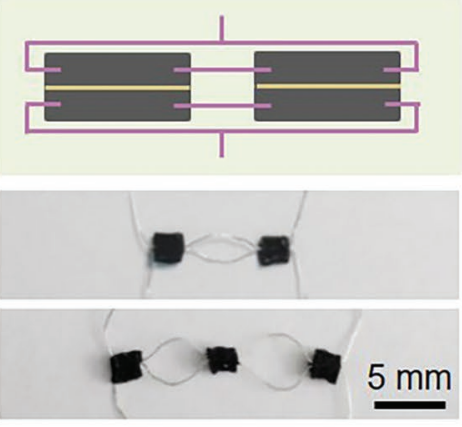

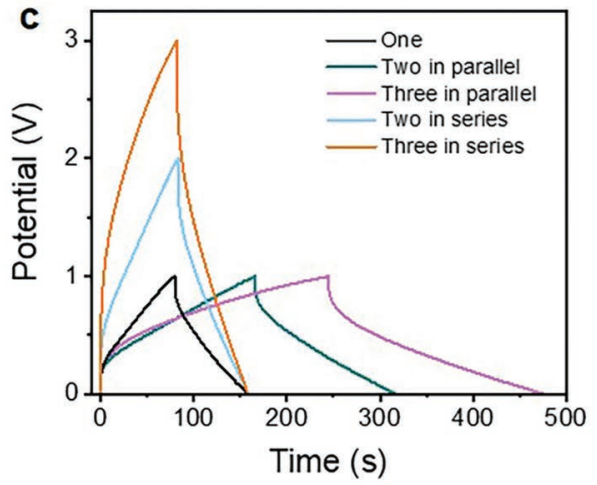

d
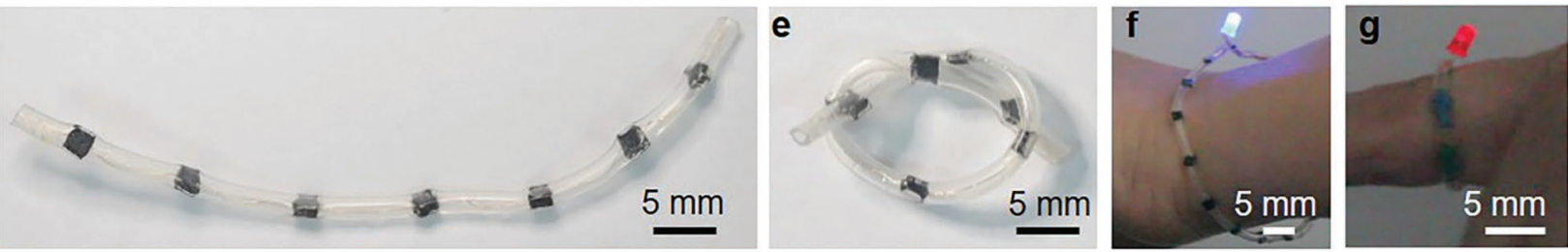

h
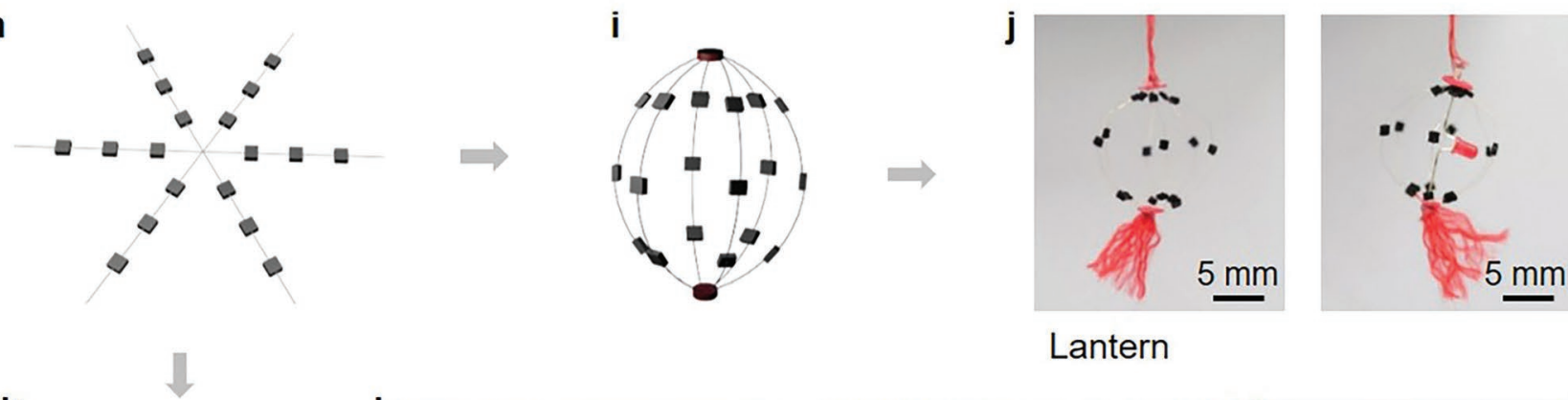

k

Lantern
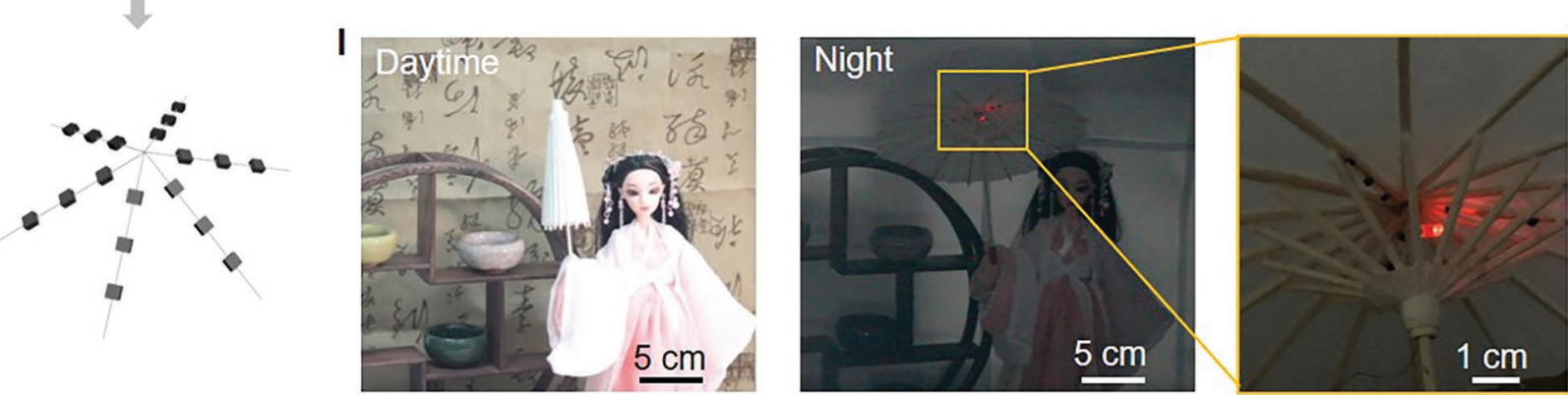

Figure 5. Integration of $\mathrm{CmSCs}$. a) Schematic diagram and photographs of two and three devices in series connection. b) Schematic diagram and photographs of two and three devices in parallel connection. c) GCD curves of single, two, and three devices in series or parallel connection. d) Photographs of seven devices in series connection, which could be packaged with transparent heat shrinkable tube and e) bent optionally. f) Photograph of a blue LED powered by an I-CmSC bracelet. g) A red LED powered by an I-CmSC ring. h) Schematic diagram of I-CmSC in hexagram form. i) Schematic diagram of I-CmSC ball transformed from hexagram I-CmSC. j) Optical images of I-CmSC lantern in Chinese-style. k) Schematic diagram of I-CmSC in cone form transformed from hexagram I-CmSC. I) I-CmSC positioned on a diminutive umbrella, which lights an LED when the umbrella opens.

the whole device volume to achieve the high-performance capacitive behavior. The SSA strategy is universal for many hydrogel-typed electrode materials to construct compact and high-performance $\mathrm{mSCs}$, possessing great potential in application as flexible and transformable power sources.

\section{Experimental Section}

Preparation of rGO Hydrogel: The GO aqueous dispersion used here was prepared by the modified Hummers method reported in the previous paper. ${ }^{[4]]}$ A GO dispersion $\left(60 \mathrm{~mL}, 2 \mathrm{mg} \mathrm{mL}^{-1}\right)$ was added into a Teflon-lined autoclave, and treated by a two-step hydrothermal process ( $95^{\circ} \mathrm{C}$ for $3 \mathrm{~h}$ and $180^{\circ} \mathrm{C}$ for $6 \mathrm{~h}$ subsequently) in air oven, resulting in a cylindrical rGO hydrogel.

Preparation of CmSC: The CmSC was built through simple laser cutting followed by assembling and shrinkage process. First, the rGO hydrogel was cut into slices using the computer-controlled laser cutting system. The dimension of each slice was $8 \mathrm{~mm}$ length $\times 8 \mathrm{~mm}$ width $\times 2 \mathrm{~mm}$ thickness. Then, conductive wires (e.g., gold wires) as current collector were inserted into the $\mathrm{rGO}$ electrode slice. The diameter of gold wire was $0.1 \mathrm{~mm}$. Afterward, two electrodes were assembled together with 


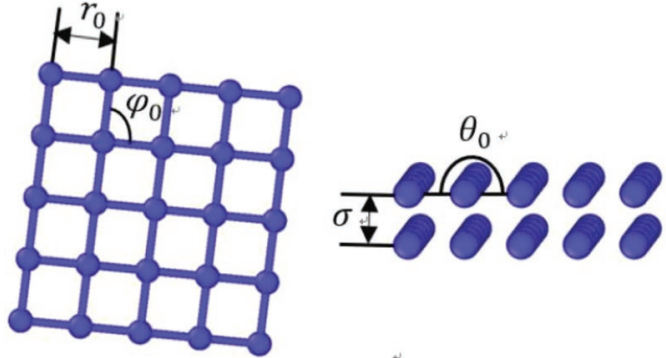

Scheme 1. Parameters illustration.

GO slurry $\left(\approx 25 \mathrm{mg} \mathrm{cm}^{-3}\right)$ as separator and binder. After placing at ambient condition $\left(25^{\circ} \mathrm{C}\right)$ for $5 \mathrm{~h}$, the device shrunk completely and the integrated supercapacitor was achieved. Furthermore, the CmSC was dipped in the gel electrolyte of polyvinyl alcohol (PVA)/LiCl and packaged with heat shrink tubing.

Preparation of Al-CmSC: First, rGO hydrogel was sculptured both the upper and lower sides based on mortise and tenon joint structure with the aid of laser cutting technology. Then, the Al-CmSC was constituted in series form, in which the intermediate electrode was shared with two single CmSCs. The detailed size of each electrode is expressed in Figure S18 (Supporting Information). After complete contraction in ambient condition, the autologous integrated device was prepared and presented as a small hard block.

Preparation of I-CmSC: I-CmSC was fabricated by $\mathrm{CmSC}$ integrating in series or parallel configuration. $\mathrm{rGO}$ hydrogel devices were connected together during hydrogel state and shrunk to a unified whole after dehydration completely.

Process and Method of MD Simulation: Calculation Model: Here, coarse grain MD simulation was conducted to uncover the mesoscale deformation mechanism during contraction process. To reproduce the porous structure, random points were seeded first, based on which Voronoi tessellation was used to generate a 3D Voronoi polyhedra, and rGO and GO sheets were randomly placed on the faces of polyhedra. By noticing $\mathrm{rGO}$ and $\mathrm{GO}$ layers had different mean void size, i.e., 12 and $6.5 \mu \mathrm{m}$ for rGO and GO layers, respectively, random points were seeded in different densities. In this way, the mesoscale structural feature could be well captured. From mechanical perspective, rGO and GO sheets had very different adhesion properties due to the functional groups on the surface of GO sheets. This basically made GO sheets bound with each other much more strongly, which was reflected in the model by assigning different values for inter-layer interaction. In general, this model well encompassed both structural and mechanical characteristics of this system.

The Model Details: In this model, each graphene sheet was depicted by $5 \times 5$ coarse grain particles, which composed a square sheet with $3 \mu \mathrm{m}$ side length and $0.1 \mu \mathrm{m}$ thickness (Scheme 1). According to Cranford and Buehler, ${ }^{[42]}$ the total energy of graphene sheets could be written as

$\phi_{\text {System }}=\phi_{T}+\phi_{\varphi}+\phi_{\theta}+\phi_{v d w}$

where the first three terms were harmonic potentials given by

$\phi_{T}=\frac{1}{2} K_{T}\left(r-r_{0}\right)^{2}, \phi_{\varphi}=\frac{1}{2} K_{\varphi}\left(\varphi-\varphi_{0}\right)^{2}, \phi_{\theta}=\frac{1}{2} K_{\theta}\left(\theta-\theta_{0}\right)^{2}$

where spring coefficients for bonds were $K_{T}=16214000 \mathrm{pg} \mathrm{\mu s}^{-2}$, and two angle spring coefficients were set to $K_{\varphi}=527625 \mathrm{pg} \mathrm{\mu m}^{2} \mu \mathrm{s}^{-2}$ and $K_{\theta}=1126 \mathrm{pg} \mu \mathrm{m}^{2} \mu \mathrm{s}^{-2}$. The equilibrium distance and angles were $r_{0}=0.75 \mu \mathrm{m}, \varphi_{0}=90^{\circ}$, and $\theta_{0}=180^{\circ}$. Besides, Lennard-Jones potential was used to delineate the van der Waals interaction between graphene sheets

$\phi_{\nu d w}=4 \varepsilon\left[(\sigma / r)^{12}-(\sigma / r)^{6}\right]$ where $\varepsilon=520 \mathrm{pg} \mu \mathrm{m}^{2} \mu \mathrm{s}^{-2}$ and the equilibrium distance $\sigma=0.1 \mu \mathrm{m}$. Same potentials and parameters were used to GO sheets, except the interaction between $\mathrm{GO}$ sheets or between $\mathrm{GO}$ sheets and $\mathrm{rGO}$ sheets was set to $\varepsilon=5200 \mathrm{pg} \mu \mathrm{m}^{2} \mu \mathrm{s}^{-2}$ (to describe the chemical adsorption due to functional groups on the surface of $G O$ sheets), which was one order larger than van der Waals interaction between graphene sheets.

In the simulation, a $30 \times 30 \times 60 \mu^{3}$ region around the $\mathrm{rGO} / \mathrm{GO}$ interface was focused (see Figure S3a, Supporting Information), and there were approximately $2000 \mathrm{rGO}$ and $2000 \mathrm{GO}$ sheets involved. Note that periodic boundary condition was adopted. To simulate the contraction process, the simulation box size was gradually reduced, and then minimization was used to stabilize the system, which would be further relaxed via enough amounts of time steps until less than $0.1 \%$ energy variation within 1000 time steps (time step was set to $0.5 \mathrm{ps}$ ).

Characterization: The morphology of the samples was investigated using a scanning electron microscope (SEM, JSM-7001F). X-ray energy disperse spectra (EDS) data were collected on TecnaiC2 20ST (T20) at an acceleration voltage of $100 \mathrm{kV}$. Compression tests were carried out using a Shimadzu AGS-X. The rGO hydrogel slices were cut by a $355 \mathrm{~nm}$ ultraviolet laser cutting system (Beijing Lagamin Laser Co., LM-UVY-5S-Y) under ambient conditions.

Electrochemical Measurements: All electrochemical measurements were performed on a $\mathrm{CHI} 760$ workstation at ambient condition. The electrochemical performance of the device was evaluated by CV measurements with scan rates from 10 to $200 \mathrm{mV} \mathrm{s}^{-1}$ and GCD profiles conducted at current densities ranging from 50 to $800 \mathrm{~mA} \mathrm{~cm}^{-3}$, with $\mathrm{PVA} / \mathrm{LiCl}$ as electrolyte. EIS was recorded in the frequency range from $100 \mathrm{kHz}$ to $0.01 \mathrm{~Hz}$ with the ac amplitude of $5 \mathrm{mV}$. Cycling stability was carried at the current density of $1.2 \mathrm{~A} \mathrm{~cm}^{-3}$. The volume capacitance was calculated based on the volume of the whole device except for the gold wire which parts were exposed.

\section{Supporting Information}

Supporting Information is available from the Wiley Online Library or from the author.

\section{Acknowledgements}

The authors acknowledge the financial support from the National Key R\&D Program of China (2017YFB1104300, 2016YFA0200200), NSFC (nos. 51673026, 51433005, 21774015, 21604003), NSFCMAECI (51861135202), NSFC-STINT (21911530143), and NSFC (grant nos. 11602272, 11972349).

\section{Conflict of Interest}

The authors declare no conflict of interest.

\section{Keywords}

high volumetric capacitance, large-scale integration, microsized supercapacitors, mortise and tenon joints, self-shrinkage assembly

Received: October 25, 2019

Revised: November 19, 2019

Published online: December 18, 2019

[1] M. Beidaghi, Y. Gogotsi, Energy Environ. Sci. 2014, 7, 867.

[2] S. M. Mirvakili, I. W. Hunter, Adv. Mater. 2017, 29, 1700671. 
[3] D. Ge, L. Yang, L. Fan, C. Zhang, X. Xiao, Y. Gogotsi, S. Yang, Nano Energy 2015, 11, 568.

[4] P. G. Bruce, B. Scrosati, J. M. Tarascon, Angew. Chem., Int. Ed. 2008, 47, 2930.

[5] M. M. Shaijumon, E. Perre, B. Daffos, P. L. Taberna, J. M. Tarascon, P. Simon, Adv. Mater. 2010, 22, 4978.

[6] K. Wang, W. Zou, B. Quan, A. Yu, H. Wu, P. Jiang, Z. Wei, Adv. Energy Mater. 2011, 1, 1068.

[7] N. Kurra, M. K. Hota, H. N. Alshareef, Nano Energy 2015, 13, 500.

[8] J. Ye, H. Tan, S. Wu, K. Ni, F. Pan, J. Liu, Z. Tao, Y. Qu, H. Ji, P. Simon, Y. Zhu, Adv. Mater. 2018, 30, 1801384

[9] M. F. El-Kady, R. B. Kaner, Nat. Commun. 2013, 4, 1475.

[10] S. Zheng, J. Ma, Z. S. Wu, F. Zhou, Y. B. He, F. Kang, H. M. Cheng, X. Bao, Energy Environ. Sci. 2018, 11, 2001.

[11] X. Shi, Z. S. Wu, J. Qin, S. Zheng, S. Wang, F. Zhou, C. Sun, X. Bao, Adv. Mater. 2017, 29, 1703034.

[12] C. J. Zhang, M. P. Kremer, A. Seral-Ascaso, S. H. Park, N. McEvoy, B. Anasori, Y. Gogotsi, V. Nicolosi, Adv. Funct. Mater. 2018, 28, 1705506.

[13] W. W. Liu, C. X. Lu, X. L. Wang, R. Y. Tay, B. K. Tay, ACS Nano 2015, 9, 1528.

[14] Y. Yue, N. Liu, Y. A. Ma, S. L. Wang, W. J. Liu, C. Luo, H. Zhang, F. Cheng, J. Y. Rao, X. K. Hu, J. Su, Y. H. Gao, ACS Nano 2018, 12, 4224

[15] N. A. Kyeremateng, T. Brousse, D. Pech, Nat. Nanotechnol. 2017, 12, 7.

[16] Q. Zhang, X. Wang, Z. Pan, J. Sun, J. Zhao, J. Zhang, C. Zhang, L. Tang, J. Luo, B. Song, Z. Zhang, W. Lu, Q. Li, Y. Zhang, Y. Yao, Nano Lett. 2017, 17, 2719.

[17] S. Bellani, E. Petroni, A. E. Del Rio Castillo, N. Curreli, B. Martín-García, R. Oropesa-Nuñez, M. Prato, F. Bonaccorso, Adv. Funct. Mater. 2019, 29, 1807659.

[18] Q. Jiang, N. Kurra, C. Xia, H. N. Alshareef, Adv. Energy Mater. 2017, 7, 1601257.

[19] Y. Y. Peng, B. Akuzum, N. Kurra, M. Q. Zhao, M. Alhabe, B. Anasori, E. C. Kumbur, H. N. Alshareef, M. D. Ger, Y. Gogotsi, Energy Environ. Sci. 2016, 9, 2847.

[20] Y. L. Shao, J. M. Li, Y. G. Li, H. Z. Wang, Q. H. Zhang, R. B. Kaner, Mater. Horiz. 2017, 4, 1145.

[21] W. Gao, N. Singh, L. Song, Z. Liu, A. L. M. Reddy, L. Ci, R. Vajtai, Q. Zhang, B. Wei, P. M. Ajayan, Nat. Nanotechnol. 2011, 6, 496.
[22] L. Li, J. Zhang, Z. Peng, Y. Li, C. Gao, Y. Ji, R. Ye, N. D. Kim, Q. Zhong, Y. Yang, H. Fei, G. Ruan, J. M. Tour, Adv. Mater. 2016, 28, 838.

[23] S. E. Moosavifard, J. Shamsi, M. K. Altafi, Z. S. Moosavifard, Chem. Commun. 2016, 52, 13140.

[24] X. Pu, M. Liu, L. Li, S. Han, X. Li, C. Jiang, C. Du, J. Luo, W. Hu, Z. L. Wang, Adv. Energy Mater. 2016, 6, 1601254.

[25] L. Yang, M. Zou, S. Wu, W. Xu, H. Wu, A. Cao, ACS Nano 2017, 11, 2944

[26] T. Gao, Z. Zhou, J. Yu, J. Zhao, G. Wang, D. Cao, B. Ding, Y. Li, Adv. Energy Mater. 2019, 9, 1802578.

[27] Y. Tao, X. Xie, W. Lv, D. M. Tang, D. Kong, Z. Huang, H. Nishihara, T. Ishii, B. Li, D. Golberg, F. Kang, T. Kyotani, Q. H. Yang, Sci. Rep. 2013, 3, 2975

[28] H. Li, Y. Tao, X. Zheng, J. Luo, F. Kang, H. M. Cheng, Q. H. Yang, Energy Environ. Sci. 2016, 9, 3135

[29] G. Qu, J. Cheng, X. Li, D. Yuan, P. Chen, X. Chen, B. Wang, H. Peng, Adv. Mater. 2016, 28, 3646

[30] W. Ma, M. Li, X. Zhou, J. Li, Y. Dong, M. Zhu, ACS Appl. Mater. Interfaces 2019, 11, 9283.

[31] X. Ma, X. Hong, L. He, L. Xu, Y. Zhang, Z. Zhu, X. Pan, J. Zhu, L. Mai, ACS Appl. Mater. Interfaces 2019, 11, 948.

[32] L. Zhang, D. DeArmond, N. T. Alvarez, R. Malik, N. Oslin, C. McConnell, P. K. Adusei, Y. Y. Hsieh, V. Shanov, Small 2017, 13, 1603114.

[33] M. Beidaghi, C. Wang, Adv. Funct. Mater. 2012, 22, 4501

[34] B. D. Boruah, A. Maji, A. Misra, ACS Appl. Mater. Interfaces 2018, $10,15864$.

[35] X. Chen, L. Qiu, J. Ren, G. Guan, H. Lin, Z. Zhang, P. Chen, Y. Wang, H. Peng, Adv. Mater. 2013, 25, 6436.

[36] Z. Li, M. Shao, L. Zhou, R. Zhang, C. Zhang, J. Han, M. Wei, D. G. Evans, X. Duan, Nano Energy 2016, 20, 294.

[37] J. Lin, C. Zhang, Z. Yan, Y. Zhu, Z. Peng, R. H. Hauge, D. Natelson, J. M. Tour, Nano Lett. 2013, 13, 72.

[38] Y. Wang, Y. Z. Zhang, D. Dubbink, J. E. ten Elshof, Nano Energy 2018, 49, 481

[39] H. Xiao, Z. S. Wu, L. Chen, F. Zhou, S. Zheng, W. Ren, H. M. Cheng, X. Bao, ACS Nano 2017, 11, 7284.

[40] J. G. Werner, G. G. Rodríguez-Calero, H. D. Abruña, U. Wiesner, Energy Environ. Sci. 2018, 11, 1261.

[41] Z. Wang, B. Lu, X. Meng, C. Zhao, L. Huang, Z. Liu, C. Yu, J. Qiu, Electrochim. Acta 2017, 252, 84

[42] S. Cranford, M. J. Buehler, Modell. Simul. Mater. Sci. Eng. 2011, 19, 054003 